\title{
Medical Images Segmentation Based on Unsupervised Algorithms: A Review
}

\author{
$1^{\text {st }}$ Revella E. A. Armya \\ Technical College of Informatics \\ Duhok Polytechnic University \\ Duhok, Iraq \\ revella.eshaya@dpu.edu.krd
}

\author{
$2^{\text {nd }}$ Adnan Mohsin Abdulazeez \\ Presidency of Duhok Polytechnic University \\ Duhok Polytechnic University \\ Duhok, Iraq \\ adnan.mohsin@dpu.edu.krd
}

https://doi.org/10.48161/qaj.v1n2a51

\begin{abstract}
Medical image segmentation plays an essential role in computer-aided diagnostic systems in various applications. Therefore, researchers are attracted to apply new algorithms for medical image processing because it is a massive investment in developing medical imaging methods such as dermatoscopy, X-rays, microscopy, ultrasound, computed tomography (CT), positron emission tomography, and magnetic resonance imaging. (Magnetic Resonance Imaging), So segmentation of medical images is considered one of the most important medical imaging processes because it extracts the field of interest from the Return on investment (ROI) through an automatic or semi-automatic process. The medical image is divided into regions based on the specific descriptions, such as tissue/organ division in medical applications for border detection, tumor detection/segmentation, and comprehensive and accurate detection. Several methods of segmentation have been proposed in the literature, but their efficacy is difficult to compare. To better address, this issue, a variety of measurement standards have been suggested to decide the consistency of the segmentation outcome. Unsupervised ranking criteria use some of the statistics in the hash score based on the original picture. The key aim of this paper is to study some literature on unsupervised algorithms (K-mean, K-medoids) and to compare the working efficiency of unsupervised algorithms with different types of medical images.
\end{abstract}

Keywords-Medical Images, Segmentation, Partition Around Medoids, K-means, Feature Selection.

\section{INTRODUCTION}

Explain here about Medical Images, which have characteristics of a different nature than standard images. As an example of an important feature, there is usually a difference in the distribution of data between the target and source domains. These variations can lead to poor results when templates are explicitly applied to the medical image field in the general image field [1]. Most medical images suffer from insufficient contrast and clarity. This results in blurry or blurred edges (low contrast) between neighboring tissues, resulting in poor segmentation, error in tissue recognition. Contrast improvement to increase visual knowledge is also essential in implementing methods for capturing objective measurements from medical photographs. [2][3].

There are several methods widely used. For example, (ultrasound, mammogram, Ct-Scan, MRI). It is using ultrasound for cancers diagnosed. Although radiography is used to measure agglomerations in routine clinical practice, it increases the visualization of invisible and tiny tumors; ultrasound is more favored because it is more accessible, more affordable, and time-consuming for patients [4]. Furthermore, the predictive radiographic value is typically tiny. Patients of benign cancers, however, undergo needless examinations. As an aid to radiography, this constraint to radiography was solved by ultrasound [5].

A common technique used to classify early-stage cancer cells is X-ray imaging. For radiologists, However, it is impossible to examine hundreds of mammograms a day. The task is time-consuming and lengthy, which results in false negatives and false positives. This artifact includes strong gravity values and is optically close to unnatural, misleading, and avoiding adoption of micro-disease-carrier zoning strategies [6][7].

One of the most widely used emergency room screening methods for patients with brain injuries or those with signs of a stroke or elevated intracranial pressure is non-contrast head $\mathrm{CT}$. The high availability and low timing of these tests make them a standard method of diagnosis [8]. In stroke patients, the principal objective of the diagnosis is to eliminate a computed tomographic hemorrhage based on its straightforward interpretation because the bleeding includes more severe and time-sensitive irregularities that can be rapidly seen in a CT scan, intracranial bleeding [9]. In patients with possible acute intracranial hemorrhage, an early interpretation of the CT scan is often crucial for evaluating neuro-operative care. Cranial fractures usually need urgent treatment when opened or stressed [10].

Methods of medical image segmentation for the interpretation of scenes and medical image processing, image-guided treatments, radiotherapy or improved radiological detection, etc., an essential processing stages in 
natural images. Segmentation of images is described formally as "the partition of an image into a set of no overlapping regions whose union is the entire image" (Haralick \& Shapiro 1992) [11]. In general, image segmentation in image processing is an essential method that separates the whole image into many regions to isolate the area of interest [12]. All of these techniques that are used in analyzing medical images are among the machine learning techniques. Detection and diagnostics in understanding medical imaging effects are now more reliable and quicker using machine learning algorithms. A fascinating field of study is machine learning. It allows concrete patterns to be derived from instances [13][14]. An unsupervised learning approach would design an ideal clustering algorithm for localization [15] the Medical images.

Unsupervised algorithms that cluster objects into groups increase the similarity between objects in a cluster and minimize the similarity between objects in various categories. This fascinating problem has been interesting for several years because of its many implementations [16]. Examples of unsupervised learning algorithms systems include (PAM, K-Mean).

The Euclidean distance used to measure the minimum distance between the data subjects and the focus points of the cluster [17] is the basis of the classic K-means method. A data entity is assigned to the closest medium describing the class. Consistency and pace are the main advantages of K-means to divide the number of items into known classes. But according to the stage of initialization [18], each run can have different outcomes. Additionally, because of its emphasis on Euclidean distance, K-means is not ideal for all data types. For example, it would be improper to use the $\mathrm{K}$ method for mixed or categorical datasets [19]. This restriction is also valid for binary data, the most basic form of categorical data [20]. In comparison, another threshold is exposure to outliers [21].

K-medoids have been suggested as a potential alternative in the past [18]. These issues need to be answered. The $\mathrm{K}$ medoids method relies on the array of data items in a specified class as a class average. However, the critical distinction is that the former is dependent on the average, while the latter relies on the median as a representation of the cluster [22].

Many advancements in the application of machine learning [23][24][25] to the understanding of medical imaging tasks have been seen in recent years, With strong evidence that deep learning can conduct a range of medical imaging activities, including the identification and classification of diabetic retinopathy. [26] and the classification of skin lesions. They are either benign or malignant [27] with an equal specificity to that of medical practitioners. Deep learning algorithm was also learned to recognize radiological photos anomalies, for example, chest images [23][28], chest tomography [29][30], chest tomography [29][30], In addition to localizing disease pattern determination or Anatomical volumes [31][32] by segmentation algorithms, and computed tomography [24][33] by classification algorithms.

A feature is an actual observable property of the process being studied Today [34]. As a result of technical development that contributes to tremendous data processing power, the amount of features used in a process is growing.
All of the metrics are no longer true as the number of features grows, where selections need to be made. The topic of feature selection is to gain attention from researchers concerned with the discriminant analysis and classification problems. Likewise, we also encounter many possible features in many other applications that can be used for further classification activities. Therefore, it is generally a false choice to use all the traits we have for classification. Knowing the attributes that can lead to the highest classification outcome is also important. Therefore, the need for a limited number of discriminatory features is varied; it is cheaper and simpler to deal with the curse of dimensionality to collect a reduced collection of meaningful predictors, decrease computational pressure, allow us to understand the domain better, and boost the classification algorithm efficiency [35][36][37].

This paper mainly aims to study such literature on unsupervised algorithms (K-mean, K-medoids) and equate the operating efficiency of unsupervised algorithms with various types of medical images.

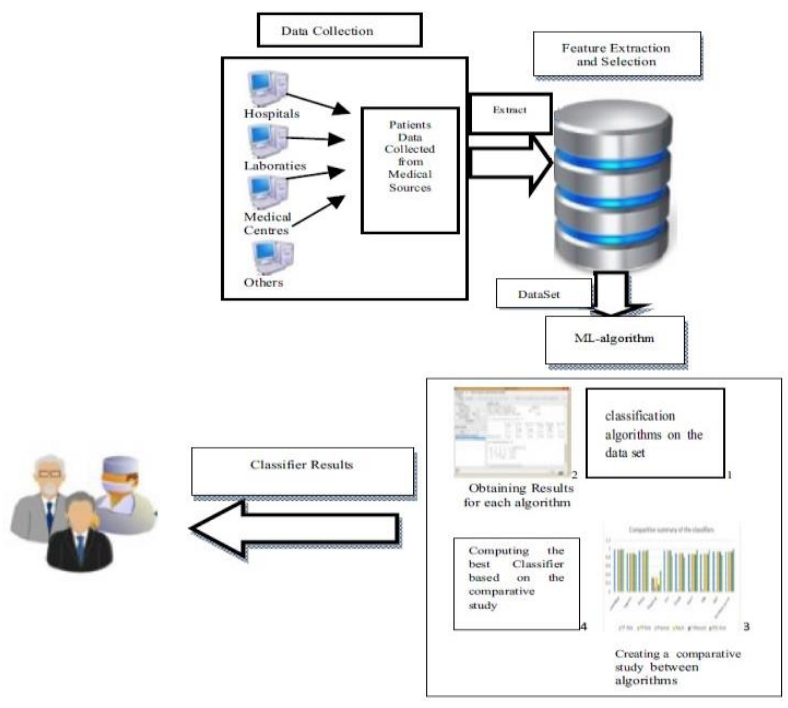

Fig. 1. Analysis of Medical Images using Machine Learning Tools [30]

\section{MEDICAL IMAGES}

Researchers developed systems for automated processing until it became possible to view medical images and transfer them to a computer. A series of low-level pixel processing (edge line detector filters and area growth) and mathematical modeling (composition lines, circles, and ellipses) were first carried out during a medical image analysis in the 1970s to construct a composite system-based basis to solve a specific challenge. A range of "if-else" statements popular in AI is close to expert frameworks around the same period. These structures have been known as GOFII (Old Fashioned Artificial Intelligence). In the late 1990s, supervised methods used in analyzing medical images through training data to construct a method were popular [38].

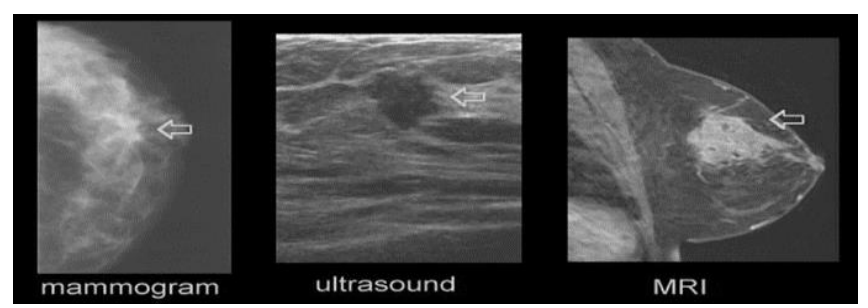

Fig. 2. Medical images: Mammogram - Ultrasound - MRI [43] 


\section{A. Ultrasound Images}

Experts use ultrasound in image inspection systems because they produce high-frequency sound waves that permeate the human body, and as the waves bounce off the boundary tissue of the human body, distinctive echoes will be created, which the computer uses to produce the image. Where some palpable lesions (lumps) can be evaluated accurately to identify cysts in the human body, especially in the breast, and thus can help the radiologist to detect lumps [39][40].
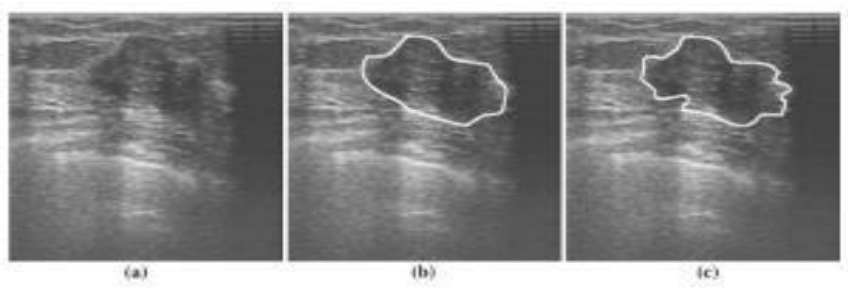

Fig. 3. Transformation based for Ultrasound Segmentation: (a) The original Ultrasound, (b) Manual Delineation, and (c) Result of Segmentation [41]

\section{B. Mammogram Images}

In the current studies, mammogram has been focused on segmentation. These studies are often categorized according to their segmentation techniques into five groups, namely, threshold technique, conformation-based, active circumference, area elevation, and tissue-based [42]. In radiography, precise delimitation poses two key issues. Owing to the unbalanced tension on the tissues during acquisition, the comparison of the proximal area steadily decreases. During digitization, boundary illumination is further decreased in optical radiographs due to additional noise [43].
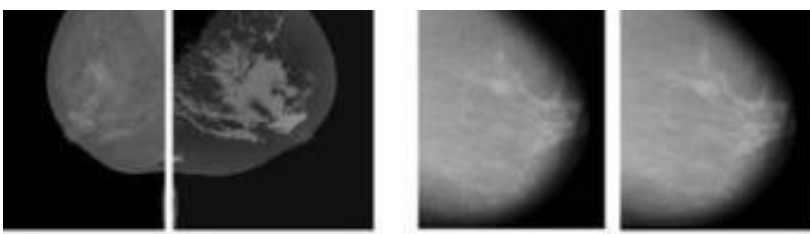

Fig. 4. Mammogram preprocessing for images from IN-breast and MIAS databases [43]

\section{CT-Scan}

CT scan is commonly used to evaluate patients with an acute head injury who need hospital admission and examination. Imaging allows an early assessment of the magnitude of the damage, which can be accessed easily using new, readily available, high-resolution multi-detector scanners. In anxious patients and those that are ventilated but dysfunctional because of extreme trauma, the short period of imaging and the ease of retrieval are of great benefit. Images slices that are degraded from moving objects can be repeated quickly. Imaging data may be visualized using brain or bone comparison windows to show bone damage and intracranial anatomy and reconstructed into 3D CT data sets [8].

\section{Magnetic Resonance Image (MRI)}

The (MRI) data is a series of time back frame sequences. Because of the intense magnetic reaction and other external factors of the head movement, it is very noisy when used for brain imagery. For testing purposes, spatial filters will be used to correct the MRI results. MRI photos are taken remotely from the free BRATS website for the investigation of brain tumors. A selection of different disease images for study and analysis is accessible in the BRATS database [44].
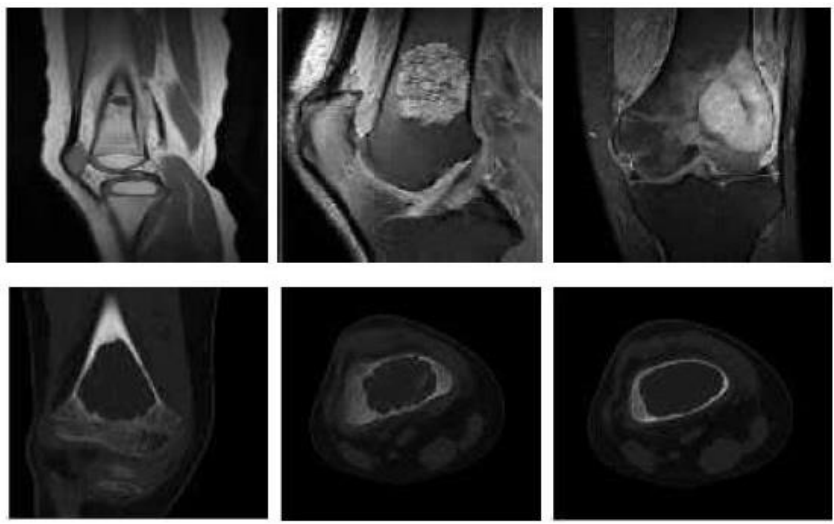

Fig. 5. MRI in the first row \& CT scan in the second row [45]

\section{UNSUPERVISED ALGORITHMS}

Clustering is an unsupervised classification, grouping data items of a very similar standard into the same group, whereas other objects of a different nature fall into separate categories-clustering algorithms. E.g., hierarchical clustering: Figure 3, are categorized into two central hierarchical clusterings or clustering. E.g., non-hierarchical clustering figures 6-7; K-means and K-medoids are typical of non-hierarchical clustering algorithms (PAM) [46].
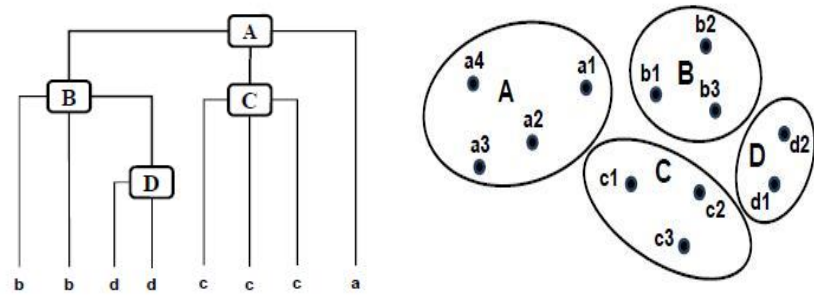

Fig. 6. Hierarchal Clustering [47] Fig. 7. Partitioning Clustering [47]

\section{A. Partition Around Medoids (PAM)}

The PAM algorithm is a flexible and accurate algorithm for clustering [48]. Kaufman and Rousseau were the first to suggest the PAM algorithm [49]. This is one of the KMedoids strategies most useful. And the noise is stable, and the algorithm is an outlier. Medoids are not part of the continuous compilation of data by way of which they are part. A medoid is static, where the mean discrepancy between a medoid and other points in the data set is marginal [50][51]. The concept for the PAM algorithm is that the data sets will be separated into $\mathrm{k}$ subsets, and the cluster medoids will then be improved iteratively to minimize the targets. The Pam algorithm is chosen the number of $\mathrm{k}$ partitions in which the random k partitions are performed as Medoids. At each point, the exchange of current medoids for non-medoid items improving cluster efficiency is analyzed for non-medoid items from the datasets. The outcome is calculated in the cluster by dividing all distances from non-medoid artifacts to medoids [52].

The PAM algorithm is more robust than the K-mean because the outlier or specific other extreme values have a more negligible effect on the medoids than the mean value [53]. HOWEVER, the PAM algorithm has some limitation 
that is normal to K-mean; it considers all characteristics similarly, for instance, regardless of their actual relevance [54]. As a result of technological development, the calculation of features used in the process is growing. The characteristics in real-world data have varying degrees of significance, and they can vary in reasonable degrees even within related characteristics. The distance estimation for characteristics has a crucial effect on the methods of classification, where small distance patterns in the space of characteristics are identical. The distance equations have their power and weakness, such that we can use the distance metric that applies to the application requires. When implemented with clustering algorithms, the typical Mahalanobis distance exhibits good performance but has some drawbacks [35][55].

$\mathrm{K}$-Medoids is a classic grouping stratification technique that combines the n-object dataset into previously known ksets. A helpful tool is a silhouette to define $\mathrm{k}$. As compared to k-mean, it is more potent for outliers and noise. Medoids can be defined as a mass object in which the average variation is negligible with all objects in the block, i.e., In the stated data set, this is the focal point [16].

The k-medoids algorithm starts with the computation of $\mathrm{K}$ medoids and uses some distance metric to assign each object of the dataset to the closest medoids. K-medoids then measures the swap cost of swapping Pi and Medoid Mi items as follows.

$$
\operatorname{COST}_{P M}=\sum_{M_{i}} \sum_{p_{i} \in m_{i}}\left|P_{i}-M_{i}\right|
$$

The algorithm takes the following steps when this cost decreases to a defined threshold-Datapoint $\mathrm{P}$ for each medoid $\mathrm{M}$, such as $\mathrm{P} \neq \mathrm{M}$.

\section{- $\quad K$-MEDOID ALGORITHM}

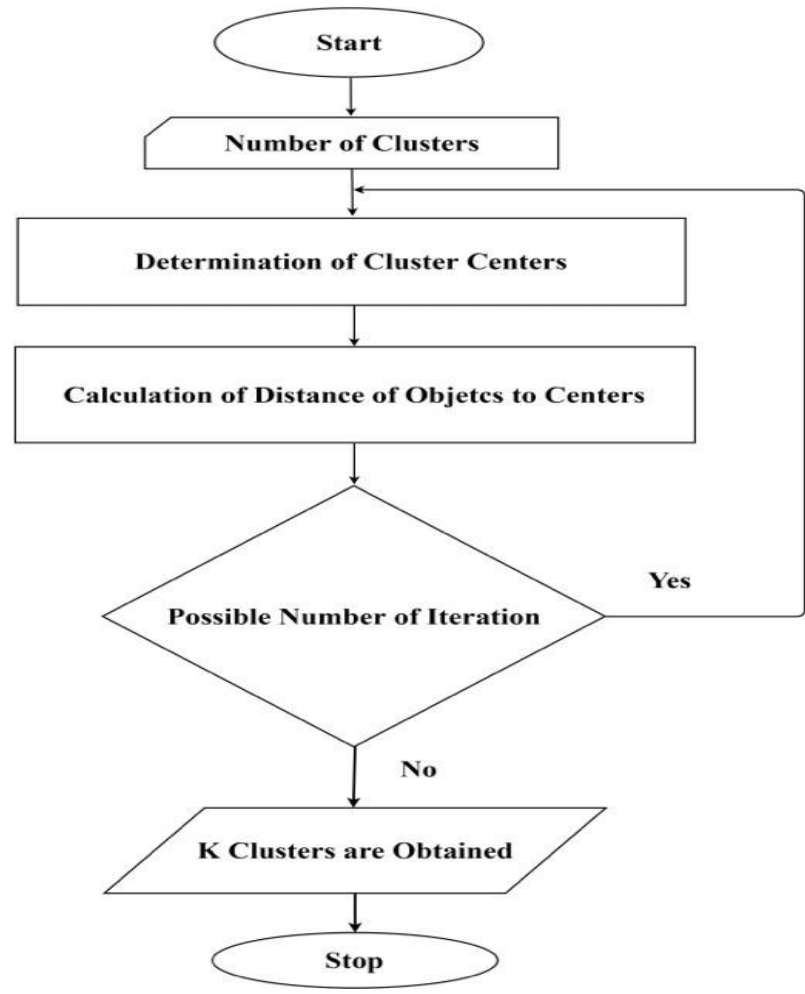

Fig. 8. Flowchart of K-medoids [3]

\section{B. K-means}

One of the fast, unattended learning algorithms that solve the widespread problem of clusters. The approach is used to identify data set by such prior fixed clusters in a straightforward and fast way (assuming k clusters) [56]. The algorithm is susceptible to the initial cluster centers that are chosen at random. To reduce this effect, the k-means algorithm can be performed many times. K-Means is an easyto-use algorithm in multiple trouble areas and is an excellent alternative to use random data points. One of the most popular approaches to solve the $\mathrm{k}$-means problem is to find a minimum solution locally centered on a basic iterative system [57][58][59][60].

$\mathrm{K}$-means are reduced by the square error difference between the cluster mean and the data points in the cluster. If we have any n-dimensional data points that the customer needs to group into a k-number of clusters with $\mu \mathrm{k}$ as the average of that cluster, then $\mathrm{k}$-means are defined as the knumber of clusters [61].

$$
\left[\sum_{k=1}^{k} \sum_{x \in c_{k}}\left\|x_{i}-u_{k}\right\|^{2}\right]
$$

Where $x i$ is a data point set consisting of $I=1,2,3, . ., n$, to be grouped into a cluster of clusters comprising $\mathrm{k}=1,2,3$, ...., $\mathrm{k}, \mathrm{K}$-means are assigning patterns to the originally partitioned $\mathrm{k}$ clusters to minimize the squared error [61]. In the case of membership of non-decisive clusters, k-means will proceed to replicate the following steps.

1) Add each pattern to its closest cluster and create new partitions.

2) New cluster average estimation.

A distant metric is another significant parameter required by k-means. Usually, for Euclidean distance metrics, kmeans measure the root of square discrepancies between object coordinates. It determines the Euclidean distance as follows:

$$
\operatorname{distance}(x, y)=\sqrt{\sum_{i=0}^{n}\left(X_{i}-Y_{i}\right)}
$$

Besides Euclidean distance, the same description is available for Manhattan distance and Minkowski distance metrics. The actual difference between the points of a pair of objects is determined by Manhattan distance as follows:

$$
\operatorname{distance}(x, y)=\left|X_{i}-Y_{i}\right|
$$

The generalization of the distances between Euclidean and Manhattan is the distance between Minkowski. Ordinal and quantitative factors should be used. The distance from Minkowski is represented in equation 4.

$$
\operatorname{distance}(x, y)=\left[\sum_{i=1}^{n}\left\|x_{i}-x_{i}\right\|^{\frac{1}{p}}\right]^{\frac{1}{p}}
$$


- $\quad K-M E D O I D ~ A L G O R I T H M$

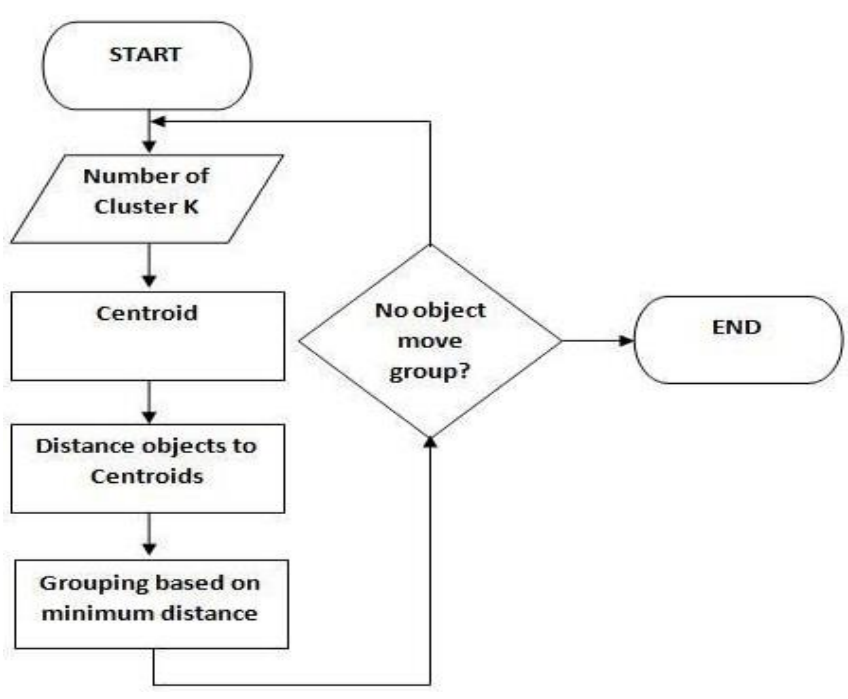

Fig. 9. Flowchart of K-means [3]

\section{RELATED WORK}

S. Hansen et al. [62] proposed an unsupervised supervoxel-based clustering method for hybrid PET/MRI lung tumor segmentation through patients. The procedure consists of two steps: first, a set of supervoxel PET/MRI features representing each patient. The data points for all cases are then translated into tumor and non-tumor supervoxels at the population level. The suggested system is assessed based on scans of 18 patients with a total of 19 nonsmall cell lung cancer tumors and evaluated concerning manual descriptions given by clinicians. Inside the structure, studies research the efficiency of many widely used clustering algorithms and evaluate (1) the impact of tumor size, (2) segmentation errors, (3) the advantages of clustering across patients, and (4) the robustness of noise.

C. Series [63] Explained that Alzheimer's is a common type of neurodegenerative disorder, such as advanced neurofibrillary tangles and amyloid plaque, characterized by weakened brain cells. The shrinking of the hippocampus part of the brain is one of the physical symptoms of Alzheimer's disease. The hippocampus is the smallest portion of the brain used to keep memories. Magnetic Resonance Imaging (MRI) is a non-invasive method for diagnosing Alzheimer's disease that helps patients with Alzheimer's disease determine brain activity. In this study, the technique K-Means Clustering and Watershed is used for fragments of hippocampus areas, which are part of the brain impacted by Alzheimer's disease. In this study, the threshold value is contrasted with the number of white pixels in videos. The analysis data were focused on a coronal slice picture of the open-access sample (OASIS) archive. Our research results in the two clusters KMeans and Watershed diagnose Alzheimer's disease in the hippocampus area.

L. Rundo et al. [64] Used the unattended Fuzzy C-means clustering (FCM) techniques in a two-stage computing system. An automatic subdivision of all three tissue types was used. It turns out that no new plan has yet answered this problem. For evaluation utilizing overlap and distance-based measurements, tissue-specific picture subdivision (pelvic/ovarian and vomiting disorders) and RCCD-data sets were analyzed. The findings revealed that the two-stage segmentation method outperformed the standard segmentation methodology for all the tasks of subdivision tests: set a multi-threshold, a technique for Otsu, and a Kmean clustering algorithm for automated category number preference inference. Studies also found that incorporating spatial knowledge into the FCM algorithm typically yields more accurate segmentation performance, whereas variants of the FCM kernel are not helpful. In both the high-density and the low-density elements starting with $81.94 \pm 4.76$, $83.43 \pm 3.81$, for the sub-segmentation tasks tested, the best spatial FCM configuration obtained an average dice-like coefficient value.

P. G. YILMAZ et al. [65] investigated two separate follicle detection methods for polycystic ovary syndrome. The first technique consists of filtering noise, modifying the contrast, binarizing, and morphological processes. Median Filter, Average Filter, Gaussian Filter, and Wiener Filter for noise reduction were used for this technique, and then histogram equalization and adaptive thresholding were checked. Gaussian Filter and Wavelet Transform were chosen for the second approach for noise reduction, and clustering of k-means and morphological operations were applied to the images. Follicles were found with the Canny Edge Detection algorithm in the segmentation process performed for both techniques. The False Acceptance Rate (FAR) and the False Rejection Rate (FRR) were used to determine the consistency of the resurrection.

I. H. Aboughaleb et al. [66] Hyperspectral imaging (HSI) has been used to analyze HSI data for breast cancer identification with advanced image processing and pattern recognition. In both malignant and natural tissues, spectral signatures have been excavated and assessed. Different responses to light transmission and absorption, especially reflection across the spectrum, are provided when modifying the optical properties of breast tissue ex vivo. Silent K-means a clustering algorithm for the hyperspectral data classification to test and detect cancerous tissue. This technique has been used to classify ex vivo breast cancer. Spatial, spectral maps have been developed to determine variations in the reflective characteristics of malignant tissue and normal tissue.

P. K. B. Rangaiah et al. [67] Centered on permittivity calculation and image processing, this study used two strategies for classifying human burnt skin. The first approach uses an open-end coaxial probe technique for the sectoral calculation of permittivity (dielectric profiling, DEP). Secondly, the color variation of the burnt skin sample is analyzed by changing the picture. To analyze and assess results, statistics research is performed using techniques such as Variance Analysis (ANOVA) k-means. The experimental data were classified into five categories based on media distribution (dielectric profiles) and centroids as part of the classification (color profiles). The color image can be transformed into a gray image, and a single-dimensional array resized. Furthermore, the evaluation is carried out on a force scale, various center values, and a study of silhouettes. The subsequent clustering results can be used to combine the color variation of human skin burned to correlate Dielectric proprieties. To measure the level of the flame.

H. Tai et al. [68] purposed to establish a novel method of attenuation correction based on adaptive clustering of $\mathrm{K}$ means. In order to enhance the discernment of these signals, it was possible to adjust $\mathrm{GH}$ filters by using a side-moving window technique. To prevent information leakage, the 
signal separated from the same filter is then paired with overlap-adding technology. Using a 256 US Vera Sonics scanner with linear transducer L11-4v, experimental data has been collected. Data from. In vivo demonstrate that the US. $\mathrm{H}$-scan imaging will configure the $\mathrm{GH}$ kernel by adaptive mitigation and balance the emerging attenuation spectrum (i.e., high-frequency shift). This process creates the $\mathrm{H}$-scan US images and global attenuation correction strategies. Overall, it increases the ability of US imaging to measure the scale of acoustic dispersions through the imagery of diverse tissues for the characterization of tissues and improves its clinical use.

R. Agrawal et al. [69] proposed a hybrid approach through the combination of median filter, $\mathrm{k}$ means clustering, Sobel edge identification, and morphological operations for brain lesion segmentation in various imaging modes. The median filter is a significant step in planning and collects impulsive noise from brain pictures, followed by k-means, Sobel boundary detection, and morphological processing. Regular data sets use output measures such as precise segmentation and runtime to verify the accuracy of the proposed automated method. Compared with a manual delineation by an expert radiologist, the approach suggested achieves a high precision of 94 percent. Furthermore, by expert delimitation using ANOVA and the correlations coefficient, high meaning values of 0,986 and 1 , respectively, were achieved using a statistical significance test among lesions segmented by the procedure.

D. Borys et al. [70] tested and implemented a few clustering-based, and they concentrated primarily on algorithms related to k-means to test and compare their accuracy. The authors have chosen the k-means algorithm, the k-medoids clustering, and the fuzzy C-means (FCM) approach in this group. The findings were verified using the gold standard of the anatomical and emission tomography data collection, co-registered with all methods. A Jaccard index has been used to measure the numerical values of both datasets. In clinical practice (standardized uptake value SUV with 2.4 threshare, which is a standard normal used) and with previously applied and validated procedures, a standard segmentation algorithm was compared based on a given threshold (including game-theoretical algorithm). The results for both methods were very similar, comparable to the SUV 2.5 limit, but worse than the game-theoretical approach. They were very close.

W. D. Kadhim et al. [45] used in this work on six MRI and CT scan images with various cluster numbers The Kmeans algorithm. The delicate tumor areas were effectively extracted after extraction, in addition to other morphological operations such as opening and dilation. Segmentation is one of the most critical facets of the production of medical images. In the segmentation of the camera, the optical image is one of many pixel sets. Magnetic resonance imaging, MRI, and CT scanning are highly efficient imaging methods for the non-invasive study of the body's internal physiological structures. One of the most severe disorders is a bone tumor, and so it is a pressing need to distinguish species from the tumor areas. Radiologist delineations and the relative ratio of deviations between (0.63-1.75) percent and (0.34-1.51) percent were observed in the MRI scan image and the average surface area in the actual tumor areas. This result shows the high-quality performance of the classification-based segmentation approach.
N. K. Student [71] shown that during brain/skull research regarding the position and identification of a brain tumor, the primary imagery tool is magnetic resonance imaging (MRI). Brain tumor diagnosis is an essential medical diagnosis technique. The brain MRI images display a complex brain cell network with osseous structures and assumed solid growth. A splitting process is, therefore, necessary to extract growth. In the original K-mean algorithm, the number of groups is determined by the user, i.e., user input. However, using the K-Means self-assembly algorithm to detect brain tumors with precision and limited execution time, this limitation is overcome. The Sobel edge detection system is used to separate the edges of the segmented brain tumor from the surrounding area. The number of groups is determined in the self-adaptive k-means group by calculating the peaks in the graph. To approximate its size and position, the portion divided into binary image formats is then processed. The gray version is used to obtain the compositional and chromatic characteristics of the essence of growth analysis. The final segmented fraction is added to the algorithm to estimate the tumor region by volume and estimate the circumference.

P. Sarker et al. [72] analyzed multiple morphological processing used to extract the lung $\mathrm{CT}$ image from the background, noises, and airways. Then, k-means clustering segmentation-dependent algorithms are used to identify lung tumors. Centered on the Tumor Nodule Metastasis (TNM) classification indicated by the Tumor Nodule Metastasis (TNM) classification, volumetric analysis of lung nodules was performed by the World Health Organisation to predict tumor stages (WHO). TNM is used to detect lung cancer in its quantities, spread, and metastases. A dataset called SPIEAAPM Lung CT Challenge was used to separate lung tumors from Washington University, St. Louis, with 22,489 CT images of 70 patients. The proposed 3D CT segmentation and classification method provides enhanced 95.68 percent accuracy with the correct recognition and simulation of the size, shape, and location of the variant, reduced measurement time, and the correct classification of the tumor point.

S. Yin et al. [73] demonstrated that when performing multilevel gray image segmentation, the researcher shows the adoption of the entropy of the fuzzy $\mathrm{C}$-section to find optimal thresholds. There are usually two limitations to the existing ambiguous partition entropy approaches, i.e., The C-section number has to be manually calibrated for various inputs, and only gray images can be processed using the processes. The essential step in the algorithm is the two-level segmentation factor to overcome these two shortcomings, using binary graph reductions to improve the entropy and the smoothness of the Gaussian partition. Multilevel images are segmented hierarchically by continuously applying the two-level segmentation factor: beginning with the color image data, the algorithm selects the color channel that can best divide a paired image into two labels. The quantitative studies carried out by regular grayscale images and the Berkeley Segmentation database demonstrate that this methodology is close to traditional MSM approaches, but has the advantage of being unsupervised, efficient, and easy to incorporate. The experimental results show that the hierarchical segmentation diagram presented can separate grayscale and color images efficiently.

J. Yu, D. Huang et al. [74] proposed a method for image segmentation in this work, based on automatic coding and hierarchical clustering algorithm, to deal with fragmentation 
in an uncontrolled way. More precisely, there are two stages to this suggested method: preparation and segmentation. In the training step, using a Stacked DE noising Auto-encoder (SDA), and divide the image samples into non-overlapping spots and extract deep-level representations of corrections, then perform unsupervised K-clusters and hierarchy on representations of this feature and establish an index tree structure. It achieves an arbitrary hashed image in the split step based on the structure of the index tree. However, this unregulated method has shown that it benefits relative to traditional uncontrolled segmentation strategies using sample images. Empirical assessments on many benchmark data sets show that the algorithm outperforms several other approaches in both time efficiency accuracy.

A. Dharmarajan et al. [75] helped the oncologist decide while reducing the algorithm's implementation time, and it also advanced applications for medical treatment. For the study of lung cancer results, this is very suitable for selecting a mass production algorithm. Clustering is an essential data processing technique used to classify diseases in many fields, including medical diagnosis. It is the process of data aggregation by identifying correlations between the data based on its characteristics to get to know it. In this research analysis, the efficiency of clustering algorithms over their computational time was found using lung cancer data. Computational measures have been used to extract outcomes and compare the performance of algorithms given a small range of features of data on lung cancer. The utility of both algorithms was assessed based on the results obtained from this approach by choosing k-Means and k-Mediods segmentation-based clustering algorithms to analyze lung cancer data. The best output of the algorithm is reported for the chosen data definition.

A. A. Pravitasari et al. [76] analyzed The 3D rendering of the MRI images. Using a Gaussian mixture model (GMM) with maximization of projections as an enhancement technique, the tumor area was segmented as a Region of Interest (ROI) in the MRI image. These pair segmentation strategies showed substantial improvements from the sagittal, coronal, and axial viewpoints as a consistent boundary of the tumor region to distinguish from the unaffected portion of the brain and the approximate tumor volume. Also, these findings were successfully visualized with an average volume of $749 \mathrm{~mm} 3$ for the 3D construction of the tumor site on the left side of the patient's brain.

M. Kalra et al. [77] validated the developed approach to medical imagery. By adding both synthetic and actual datasets to the algorithm. And to test the algorithm's ability to diagnose complex real-world illnesses, such as brain cancers, pulmonary tuberculosis, and skin lesion melanoma. Observing and evaluating multimodal medical images via creating an unsupervised machine learning algorithm based on the finite inverted mixture model's online variable inference. Data mining is a broad field of study involving the detection of trends and the retrieval of characteristics that are implemented in different critical areas. Especially the clinical hand, which reported a quest for evidence to support clinicians in early identification, diagnosis, and disease prevention. In multimodal clinical image processing, developments in statistical approaches have contributed to the introduction of machine learning.

K. Atrey et al. [78] planned to use a multimodal method to identify and confirm photographs of breast cancer by incorporating both ultrasound and mammography. Besides, the authors studying a particular segmentation technique that can use a two-method procedure to detect breast lesions reliably. Three common techniques have been validated and evaluated for the identification of lesions using ultrasound and mammography, for example, Means Fuzzy-c (FCM), Means K (KM), and DPSO DPSO (Darwinian Particle Swarm Improvement). The proposed dual model for detecting breast cancer was evaluated with performance metrics such as the breast mass lesion region, Jaccard Index (JI), and Dice Similarity Coefficient (DSC). Results showed that FCM surpassed other images in ultrasound and JI mammography: the highest mean (0.748) and lowered SD (0.124), DSC: highest average (0.851), and lowest SD (0.851) (0.851). (0.097). Hash accuracy: the lowest average SDD $(0,92)(0.074)$.

TABLE I. A COMPARISON OF THE WORKING EFFICIENCY

\begin{tabular}{|c|c|c|c|c|c|}
\hline \multirow{2}{*}{ Ref. } & \multirow{2}{*}{ Year } & \multicolumn{4}{|c|}{ Working Efficiency Of Unsupervised Algorithms With Different Types Of Medical Images } \\
\hline & & Methods & Datasets & Feature Selection & Results \\
\hline$[62]$ & 2021 & $\begin{array}{l}\text { Determine a set of superoxel PET / } \\
\text { MRI features by each patient and } \\
\text { convert data points and classify them } \\
\text { into ultrafast and non-neoplastic } \\
\text { neoplasms at a different level. }\end{array}$ & $\begin{array}{c}\text { Hybrid lung tumor in PET \& } \\
\text { MRI }\end{array}$ & $\begin{array}{c}\text { unsupervised } \\
\text { algorithms super } \\
\text { voxel (K-means, } \\
\text { Hierarchical E-M-C, } \\
\text { Spectral) }\end{array}$ & $\begin{array}{l}\text { Noise stability in spectral clustering and } \\
\text { segmentation of PET / MRI tumors with A } \\
\text { small number of incomplete tumors and a } \\
\text { minimal number of false positives. }\end{array}$ \\
\hline$[63]$ & 2021 & $\begin{array}{l}\text { Diagnosis of Alzheimer's disease by } \\
\text { analyzing and comparing the } \\
\text { threshold value and the number of } \\
\text { white pixels in the images. }\end{array}$ & MRI of an Alzheimer's patient & $\begin{array}{l}\text { Using K-Means } \\
\text { Clustering \& } \\
\text { Watershed }\end{array}$ & $\begin{array}{c}\text { Hippocampal region segmentation for } \\
\text { Alzheimer's disease diagnosis based on the } \\
\text { K-Means Clustering and watershed } \\
\text { approach }\end{array}$ \\
\hline [45] & 2020 & $\begin{array}{l}\text { Effectively excise the exact tumor } \\
\text { areas with MRI and CT scans with } \\
\text { different cluster numbers. }\end{array}$ & MRI images \& CT-scan & $\begin{array}{l}\text { K-mean clustering } \\
\text { algorithm }\end{array}$ & $\begin{array}{c}\text { Relative differences between }(0.63-1.75) \\
\text { percent for MRI images and }(0.34-1.51 \\
\text { percent) for CT images and measured } \\
\text { surface areas for divided tumor areas }\end{array}$ \\
\hline$[64]$ & 2020 & $\begin{array}{l}\text { Automated tissue segmentation based } \\
\text { on Fuzzy C-Means Clustering } \\
\text { Community unregulated techniques }\end{array}$ & $\begin{array}{l}\text { CT datasets of ovarian (pelvic/ } \\
\text { ovarian \& omental) cancer and } \\
\text { renal cell carcinoma }\end{array}$ & $\begin{array}{l}\text { K-mean clustering } \\
\text { algorithm }\end{array}$ & $\begin{array}{l}\text { After the spatial data was incorporated } \\
\text { into the FCM algorithm, the segmentation } \\
\text { results were more detailed. }\end{array}$ \\
\hline$[65]$ & 2020 & $\begin{array}{l}\text { Using a medium filter, Gaussian } \\
\text { filter, and a Winner filter to filter } \\
\text { noise, contrast adjustment, binary } \\
\text { and morphological operations }\end{array}$ & $\begin{array}{l}\text { Ultrasound image of Polycystic } \\
\text { Ovary Syndrome }\end{array}$ & $\begin{array}{l}\text { k-means clustering \& } \\
\text { morphological } \\
\text { operations }\end{array}$ & $\begin{array}{l}\text { Using Canny Edge Detection algorithm to } \\
\text { find follicles in the hashing process }\end{array}$ \\
\hline$[66]$ & 2020 & $\begin{array}{l}\text { Hyperspectral imaging and } \\
\text { automated image analysis for pattern }\end{array}$ & $\begin{array}{l}\text { HSI evidence for the diagnosis } \\
\text { of breast cancer }\end{array}$ & $\begin{array}{c}\text { Silent K-mean } \\
\text { clustering algorithm }\end{array}$ & spatial-spectral images were produced \\
\hline
\end{tabular}




\begin{tabular}{|c|c|c|c|c|c|}
\hline & & $\begin{array}{l}\text { detection to test and detect cancerous } \\
\text { tissue }\end{array}$ & & $\begin{array}{c}\text { for hyperspectral data } \\
\text { classification }\end{array}$ & \\
\hline$[67]$ & 2020 & $\begin{array}{l}\text { Permittance segmentary calculation } \\
\text { (dielectric profiling, DEP) through an } \\
\text { open-ended coaxial test procedure } \\
\text { and a decoloration of the brûled skin } \\
\text { by picture care. }\end{array}$ & $\begin{array}{c}\text { Medical image of human burnt } \\
\text { skin }\end{array}$ & $\begin{array}{l}\text { Variance Analysis } \\
\text { (ANOVA) \& k- } \\
\text { means }\end{array}$ & $\begin{array}{l}\text { The color difference of the burned human } \\
\text { skin using the aggregation results obtained } \\
\text { from the dielectric properties. }\end{array}$ \\
\hline [76] & 2020 & $\begin{array}{l}\text { Dividing the tumor region in the } \\
\text { MRI image as a region of interest } \\
\text { (ROI) through a Gaussian mixture } \\
\text { model (GMM) with maximizing } \\
\text { projections as an improvement } \\
\text { technique. }\end{array}$ & $\begin{array}{l}\text { Brain tumor detection through } \\
\text { MRI }\end{array}$ & $\begin{array}{l}\text { K-means cluster for } \\
\text { color-based image } \\
\text { segmentation with K- } \\
\text { medoid were both } \\
\text { used to distinguish } \\
\text { the diseases. }\end{array}$ & $\begin{array}{l}\text { 3D construction of the tumor site on the } \\
\text { left side of the patient's head with an } \\
\text { estimated } 749 \mathrm{~mm} 3 \text {. }\end{array}$ \\
\hline [77] & 2020 & $\begin{array}{l}\text { Online variable inference for limited } \\
\text { inverted admixture type, multimodal } \\
\text { medical image examination, and } \\
\text { interpretation }\end{array}$ & $\begin{array}{l}\text { MRI of brain tumors, } \\
\text { pulmonary tuberculosis, \& } \\
\text { melanoma skin lesion. }\end{array}$ & $\begin{array}{l}\text { Unsupervised } \\
\text { Algorithms }\end{array}$ & $\begin{array}{c}\text { The best predictor was generated by } \\
\text { learning the data set at the same time and } \\
\text { updating the best predictor of future data } \\
\text { at each point. }\end{array}$ \\
\hline [78] & 2020 & $\begin{array}{l}\text { Detection and confirmation of } \\
\text { photographs of breast cancer by } \\
\text { combining both ultrasound and } \\
\text { mammography }\end{array}$ & $\begin{array}{c}\text { Breast cancer images: } \\
\text { (ultrasound \& mammography) }\end{array}$ & $\begin{array}{l}\text { K-means (KM), } \\
\text { Fuzzy-C-means } \\
\text { (FCM) \& (DPSO) }\end{array}$ & $\begin{array}{l}\text { FCM outperformed those in ultrasound } \\
\text { \&mammography images in identifying the } \\
\text { lesion. }\end{array}$ \\
\hline [69] & 2018 & $\begin{array}{c}\text { Use output metrics such as } \\
\text { segmentation precision and execution } \\
\text { time to determine the consistency of } \\
\text { the proposed automated system on } \\
\text { regular datasets. }\end{array}$ & $\begin{array}{c}\text { (Ct-scan \& MRI) for brain } \\
\text { images }\end{array}$ & $\begin{array}{l}\text { median filter \& } \mathrm{k}- \\
\text { means clustering }\end{array}$ & $\begin{array}{c}\text { The statistical significance test for } \\
\text { separated lesions using the automatic } \\
\text { approach resulted in high significance } \\
\text { values of } 0.986 \text { and } 1 .\end{array}$ \\
\hline$[72]$ & 2018 & $\begin{array}{c}\text { Use multiple morphologies to extract } \\
\text { lung CT images from background, } \\
\text { noise, and airways, using the } \\
\text { SPIEAAPM Lung CT Challenge } \\
\text { dataset. }\end{array}$ & CT images for lung cancer & $\begin{array}{l}\text { k-means clustering } \\
\text { segmentation } \\
\text { dependent algorithms }\end{array}$ & $\begin{array}{c}\text { Better output with } 95.68 \% \text { accuracy in } \\
\text { accurate tumor identification, variable } \\
\text { size, shape and position visualization, less } \\
\text { calculation time, and appropriate } \\
\text { classification of tumor stages. }\end{array}$ \\
\hline
\end{tabular}

\section{COMPARISON AND DESICCATION}

This research paper focused on the segmentation of medical images based on algorithms that are not supervised. The results were satisfactory but different due to the different types of medical images. However, the algorithms were working to reduce noise to determine the differences between them and normal. Pictures. So that the diagnosis is more accurate, and from these medical images: CT, ultrasound, magnetic resonance imaging (MRI), mammography, as well as its application to various samples, tumors, and diseases.

In previous studies, we find that unsupervised algorithms worked with segmentation and analysis of ovarian cancer, breast cancer, and lung cancer with satisfactory and accurate results, as we find in multiple morphological processing to extract a lung CT image from background, noise, and airways, using a dataset called SPIEAAPM Lung CT Challenge. For lung cancer, the outcome was the best output with an accuracy of 95.68 percent inaccurate tumor identification, visualization of variable scale, form, position, less time to measure, and adequate description of tumor phases.

The variance analysis (ANOVA) focused on the permittivity (dielectric sampling, DEP) of sectoral computing using an open-ended coaxial probe technique. On processing the images of color change in the sample of burned skin and determining the degree of burn, the results were used to compare the dielectric properties with the different colorations of the human burned skin.

The outcome was spectral-spatial image generation, as with hyperspectral imagery (HSI) with advanced imaging and breast cancer sensing with advanced image processing and pattern recognition.

The hippocampal component of C-means Clustering and Watershed reported Alzheimer's disease compared with the threshold. The number of white pixels in the photographs through magnetic resonance imaging (MRI) of an Alzheimer's patient. Brain tumor detection outcomes were better with K-medoid learning strategies. They pooled learning strategies produced the best predictor by learning to collect data simultaneously and update the best predictor of future data ever.

\section{CONCLUSION}

This paper provides an unsupervised approach to medical image recording and does not involve any supervised knowledge. Where partition assessment is a significant difficulty in the study of images. E.g., approaches with recording precision by using large-scale MRI brain-data-sets for recording newer 3D images during more rapid volume orders. The model analysis illustrates that it can be stable for modular organization and can be constructed according to multiple datasets and adjusted easily for precise and runtime variations. The main objective of this paper is to review some of the research on unsupervised algorithms (K-mean, Kmedoids), and to compare the working efficiency of these algorithms with different types of medical images. This route analyzes and processing of medical images can be significantly accelerated while promoting emerging developments in learning-based documentation.

\section{REFERENCES}

[1] Z. Li, M. Dong, S. Wen, X. Hu, P. Zhou, and Z. Zeng, "CLU-CNNs: Object detection for medical images," Neurocomputing, vol. 350, pp. 53-59, 2019, doi: 10.1016/j.neucom.2019.04.028.

[2] A. Gandhamal, S. Talbar, S. Gajre, A. F. M. Hani, and D. Kumar, "Local gray level S-curve transformation - A generalized contrast enhancement technique for medical images," Comput. Biol. Med., vol. 83, pp. 120-133, 2017, doi: 10.1016/j.compbiomed.2017.03.001.

[3] P. Kumar and D. Sirohi, "Comparative analysis of FCM and HCM algorithm on Iris data set," Int. J. Comput. Appl., vol. 5, no. 2, pp. 3337, 2010, doi: 10.5120/888-1261. 
[4] D. Q. Zeebaree, H. Haron, A. M. Abdulazeez, and D. A. Zebari, "Machine learning and Region Growing for Breast Cancer Segmentation," 2019 Int. Conf. Adv. Sci. Eng. ICOASE 2019, pp. 8893, 2019, doi: 10.1109/ICOASE.2019.8723832.

[5] M. A. Mohammed, B. Al-Khateeb, A. N. Rashid, D. A. Ibrahim, M. K. Abd Ghani, and S. A. Mostafa, "Neural network and multi-fractal dimension features for breast cancer classification from ultrasound images," Comput. Electr. Eng., vol. 70, pp. 871-882, 2018, doi: 10.1016/j.compeleceng.2018.01.033.

[6] M. Mustra, M. Grgic, and R. M. Rangayyan, "Review of recent advances in segmentation of the breast boundary and the pectoral muscle in mammograms," Med. Biol. Eng. Comput., vol. 54, no. 7, pp. 1003-1024, 2016, doi: 10.1007/s11517-015-1411-7.

[7] E. E. Sterns, "DIAGNOSIS OF BREAST PROBLEMS AND THE CANCER / BIOPSY RATE," vol. 39, 1996.

[8] J. P. Coles, "Imaging after brain injury," Br. J. Anaesth., vol. 99, no. 1, pp. 49-60, 2007, doi: 10.1093/bja/aem141.

[9] W. J. Powers et al., 2018 Guidelines for the Early Management of Patients With Acute Ischemic Stroke: A Guideline for Healthcare Professionals From the American Heart Association/American Stroke Association, vol. 49, no. 3. 2018.

[10] W. K. Erly, W. G. Berger, E. Krupinski, J. F. Seeger, and J. A. Guisto, "Radiology resident evaluation of head CT scan orders in the emergency department," Am. J. Neuroradiol., vol. 23, no. 1, pp. 103107, 2002.

[11] S. Asgari Taghanaki, K. Abhishek, J. P. Cohen, J. Cohen-Adad, and G. Hamarneh, Deep semantic segmentation of natural and medical images: a review, no. 0123456789. Springer Netherlands, 2020.

[12] A. C. Study, "Segmentation of Leukemia Cells Using Clustering :," vol. 10, no. 2, pp. 39-48, 2019, doi: 10.4018/IJSE.2019070103.

[13] B. J. Erickson, P. Korfiatis, Z. Akkus, and T. L. Kline, "Machine learning for medical imaging," Radiographics, vol. 37, no. 2, pp. 505515, 2017, doi: 10.1148/rg.2017160130.

[14] D. M. Abdullah and N. S. Ahmed, "A Review of most Recent Lung Cancer Detection Techniques using Machine Learning," pp. 159-173, 2021, doi: 10.5281/zenodo.4536818.

[15] D. M. Sulaiman, A. M. Abdulazeez, H. Haron, and S. S. Sadiq, "Unsupervised Learning Approach-Based New Optimization K-Means Clustering for Finger Vein Image Localization," 2019 Int. Conf. Adv. Sci. Eng. ICOASE 2019, pp. 82-87, 2019, doi: 10.1109/ICOASE.2019.8723749.

[16] V. Pattabiraman, R. Parvathi, R. Nedunchezhian, and S. Palaniammal, "A novel spatial clustering with obstacles and facilitators constraint based on edge deduction and K-Mediods," ICCTD 2009 - 2009 Int. Conf. Comput. Technol. Dev., vol. 1, pp. 402-406, 2009, doi: 10.1109/ICCTD.2009.92.

[17] M. H. J. and P. Jian and Kamber, "Data Mining Techniques, Third Edition," p. 847, 2011.

[18] K. Singh, D. Malik, and N. Sharma, "Evolving limitations in K-means algorithm in data mining and their removal," IJCEM Int. J. Comput. Eng. Manag. ISSN, vol. 12, no. April, pp. 2230-7893, 2011, [Online]. Available: www.IJCEM.org\%5Cnwww.ijcem.org.

[19] M. H. Kondarasaiah and S. Ananda, Kinetic and mechanistic study of $\mathrm{Ru}(\mathrm{III})$-nicotinic acid complex formation by oxidation of bromamine$\mathrm{T}$ in acid solution, vol. 27, no. 1. 2004.

[20] T. Velmurugan, "A State of Art Analysis of Telecommunication Data by k-Means and k-Medoids Clustering Algorithms," J. Comput. Commun., vol. 06, no. 01, pp. 190-202, 2018, doi: 10.4236/jcc.2018.61019.

[21] C. Ordonez and S. Diego, "Clustering Binary Data Streams with Kmeans.pdf," 2003.

[22] T. Santhanam and T. Velmurugan, "Computational Complexity between K-Means and K-Medoids Clustering Algorithms for Normal and Uniform Distributions of Data Points," J. Comput. Sci., vol. 6, no. 3, pp. 363-368, 2010.

[23] X. Wang, Y. Peng, L. Lu, Z. Lu, M. Bagheri, and R. M. Summers, "ChestX-ray: Hospital-Scale Chest X-ray Database and Benchmarks on Weakly Supervised Classification and Localization of Common Thorax Diseases," Adv. Comput. Vis. Pattern Recognit., pp. 369-392, 2019, doi: 10.1007/978-3-030-13969-8_18.

[24] M. Grewal, M. M. Srivastava, P. Kumar, and S. Varadarajan, "RADnet: Radiologist level accuracy using deep learning for hemorrhage detection in CT scans," Proc. - Int. Symp. Biomed. Imaging, vol. 2018April, pp. 281-284, 2018, doi: 10.1109/ISBI.2018.8363574.

[25] D. M. Abdulqader, A. M. Abdulazeez, and D. Q. Zeebaree, "Machine learning supervised algorithms of gene selection: A review," Technol. Reports Kansai Univ., vol. 62, no. 3, pp. 233-244, 2020.

[26] V. Gulshan et al., "Development and validation of a deep learning algorithm for detection of diabetic retinopathy in retinal fundus photographs," JAMA - J. Am. Med. Assoc., vol. 316, no. 22, pp. 24022410, 2016, doi: 10.1001/jama.2016.17216.

[27] J. Ko et al., "Dermatologist-level classification of skin cancer with deep neural networks," Nature, vol. 542, no. 7639, pp. 115-118, 2017, [Online]. Available: http://dx.doi.org/10.1038/nature21056.

[28] P. Rajpurkar et al., "CheXNet: Radiologist-level pneumonia detection on chest X-rays with deep learning," arXiv, pp. 3-9, 2017.

[29] M. Anthimopoulos, S. Christodoulidis, L. Ebner, A. Christe, and S. Mougiakakou, "Lung Pattern Classification for Interstitial Lung Diseases Using a Deep Convolutional Neural Network," IEEE Trans. Med. Imaging, vol. 35, no. 5, pp. 1207-1216, 2016, doi: 10.1109/TMI.2016.2535865.

[30] J. Z. Cheng et al., "Computer-Aided Diagnosis with Deep Learning Architecture: Applications to Breast Lesions in US Images and Pulmonary Nodules in CT Scans," Sci. Rep., vol. 6, no. April, pp. 113, 2016, doi: 10.1038/srep24454.

[31] A. Prasoon, K. Petersen, C. Igel, F. Lauze, E. Dam, and M. Nielsen, "Deep feature learning for knee cartilage segmentation using a triplanar convolutional neural network," Lect. Notes Comput. Sci. (including Subser. Lect. Notes Artif. Intell. Lect. Notes Bioinformatics), vol. 8150 LNCS, no. PART 2, pp. 246-253, 2013, doi: 10.1007/978-3-64240763-5_31.

[32] J. Patravali, S. Jain, and S. Chilamkurthy, "2D-3D fully convolutional neural networks for cardiac MR segmentation," Lect. Notes Comput. Sci. (including Subser. Lect. Notes Artif. Intell. Lect. Notes Bioinformatics), vol. 10663 LNCS, pp. 130-139, 2018, doi: 10.1007/978-3-319-75541-0_14.

[33] X. W. Gao, R. Hui, and Z. Tian, "Classification of CT brain images based on deep learning networks," Comput. Methods Programs Biomed., vol. 138, pp. 49-56, 2017, doi: 10.1016/j.cmpb.2016.10.007.

[34] G. Chandrashekar and F. Sahin, "A survey on feature selection methods," Comput. Electr. Eng., vol. 40, no. 1, pp. 16-28, 2014, doi: 10.1016/j.compeleceng.2013.11.024.

[35] M. J. Masnan et al., "Understanding Mahalanobis distance criterion for feature selection," AIP Conf. Proc., vol. 1660, no. May, 2015, doi: 10.1063/1.4915708.

[36] F. A. M. Bargarai, A. M. Abdulazeez, V. M. Tiryaki, and D. Q. Zeebaree, "Management of wireless communication systems using artificial intelligence-based software defined radio," Int. J. Interact. Mob. Technol., vol. 14, no. 13, pp. 107-133, 2020, doi: 10.3991/ijim.v14i13.14211.

[37] R. Zebari, A. Abdulazeez, D. Zeebaree, D. Zebari, and J. Saeed, "A Comprehensive Review of Dimensionality Reduction Techniques for Feature Selection and Feature Extraction," J. Appl. Sci. Technol. Trends, vol. 1, no. 2, pp. 56-70, 2020, doi: 10.38094/jastt1224.

[38] G. Litjens et al., "A survey on deep learning in medical image analysis," Med. Image Anal., vol. 42, no. December 2012, pp. 60-88, 2017, doi: 10.1016/j.media.2017.07.005.

[39] M. Muhammad, D. Zeebaree, A. M. A. Brifcani, J. Saeed, and D. A. Zebari, "A Review on Region of Interest Segmentation Based on Clustering Techniques for Breast Cancer Ultrasound Images," J. Appl. Sci. Technol. Trends, vol. 1, no. 3, pp. 78-91, 2020, doi: 10.38094/jastt1328.

[40] D. Qader Zeebaree, A. Mohsin Abdulazeez, D. Asaad Zebari, H. Haron, and H. Nuzly Abdull Hamed, "Multilevel Fusion in Ultrasound for Cancer Detection based on Uniform LBP Features," Comput. Mater. Contin., vol. 66, no. 3, pp. 3363-3382, 2021, doi: 10.32604/cmc.2021.013314.

[41] R. K. Bhathena, "Fast Facts: Contraception 3rd edition," J. Obstet. Gynaecol. (Lahore),, vol. 30, no. 8, pp. 887-887, 2010, doi: 10.3109/01443615.2010.517118.

[42] D. A. Zebari, D. Q. Zeebaree, A. M. Abdulazeez, H. Haron, and H. N. A. Hamed, "Improved Threshold Based and Trainable Fully Automated Segmentation for Breast Cancer Boundary and Pectoral 
Muscle in Mammogram Images," IEEE Access, vol. 8, pp. 203097 203116, 2020, doi: 10.1109/access.2020.3036072.

[43] I. A. Lbachir, R. Es-Salhi, I. Daoudi, and S. Tallal, "A new mammogram preprocessing method for computer-aided diagnosis systems," Proc. IEEE/ACS Int. Conf. Comput. Syst. Appl. AICCSA, vol. 2017-Octob, pp. 166-171, 2018, doi: 10.1109/AICCSA.2017.40.

[44] N. K. Student, "K-Means Clustering," 2017 Int. Conf. Energy, Commun. Data Anal. Soft Comput., pp. 1861-1865, 2017.

[45] W. D. Kadhim and R. S. Abdoon, "Utilizing k-means clustering to extract bone tumor in CT scan and MRI images," J. Phys. Conf. Ser., vol. 1591, no. 1, 2020, doi: 10.1088/1742-6596/1591/1/012010.

[46] S. Gupta, S. N. Singh, and D. Kumar, "Clustering methods applied for gene expression data: A study," Proc. - 2016 2nd Int. Conf. Comput. Intell. Commun. Technol. CICT 2016, pp. 724-728, 2016, doi: 10.1109/CICT.2016.149.

[47] N. Najat and A. M. Abdulazeez, "Gene clustering with partition around mediods algorithm based on weighted and normalized mahalanobis distance," ICIIBMS 2017 - 2nd Int. Conf. Intell. Informatics Biomed. Sci., vol. 2018-Janua, pp. 140-145, 2018, doi: 10.1109/ICIIBMS.2017.8279707.

[48] L. F. Ibrahim, "Using Modified Partitioning Around Medoids Clustering Technique in Mobile Network Planning," Int. J. Comput. Sci. Issues, vol. 9, no. 6, pp. 299-308, 2012.

[49] K. L. and R. P., "Clustering by means of Medoids," Statistical Data Analysis Based on the L1 Norm and Related Methods. pp. 405-416, 1987.

[50] A. Bhat, "K-Medoids Clustering Using Partitioning Around Medoids for Performing Face Recognition," Int. J. Soft Comput. Math. Control, vol. 3, no. 3, pp. 1-12, 2014, doi: 10.14810/ijscmc.2014.3301.

[51] E. Zhou, S. Mao, M. Li, and Z. Sun, "PAM spatial clustering algorithm research based on CUDA," Int. Conf. Geoinformatics, vol. 2016-Septe, 2016, doi: 10.1109/GEOINFORMATICS.2016.7578971.

[52] L. F. Ibrahim and M. H. Al Harbi, "Using clustering technique M-PAM in mobile network planning," 12th WSEAS Int. Conf. Comput., pp. 868-873, 2008, [Online]. Available: http://gateway.webofknowledge.com/gateway/Gateway.cgi?GWVersi on $=2 \&$ SrcAuth $=$ ORCID $\&$ SrcApp $=$ OrcidOrg $\&$ DestLinkType $=$ FullRe cord\&DestApp $=$ INSPEC $\&$ KeyUT $=$ INSPEC $11029688 \&$ KeyUID $=I N$ SPEC: 11029688

[53] T. Velmurugan and T. Santhanam, "Performance Analysis Of K-Means And K- Medoids Clustering Algorithms For A Randomly Generated Data Set," Int. Conf. Syst. Cybern. Informatics, no. November, pp. 578-583, 2016, [Online]. Available: https://www.researchgate.net/publication/234136053.

[54] R. C. De Amorim and T. Fenner, "Weighting features for partition around medoids using the minkowski metric," Lect. Notes Comput. Sci. (including Subser. Lect. Notes Artif. Intell. Lect. Notes Bioinformatics), vol. 7619 LNCS, pp. 35-44, 2012, doi: 10.1007/9783-642-34156-4_5.

[55] R. C. de Amorim, "A Survey on Feature Weighting Based K-Means Algorithms," J. Classif., vol. 33, no. 2, pp. 210-242, 2016, doi: 10.1007/s00357-016-9208-4.

[56] H. S. Park, J. S. Lee, and C. H. Jun, "A k-means-like algorithm for kmedoids clustering and its performance," 36th Int. Conf. Comput. Ind. Eng. ICC IE 2006, pp. 1222-1231, 2006.

[57] D. Bhukya, S. Ramachandram, and R. Sony A L, "Performance evaluation of partition based clustering algorithms in grid environment Using design of experiments," Int. J. Rev. Comput., pp. 46-53, 2010.

[58] A. Abraham, International Symposium on Distributed Computing and Artificial Intelligence. 2011

[59] I. M. Najim Adeen, A. M. Abdulazeez, and D. Q. Zeebaree, "Systematic review of unsupervised genomic clustering algorithms techniques for high dimensional datasets," Technol. Reports Kansai Univ., vol. 62, no. 3, pp. 355-374, 2020.

[60] D. Q. Zeebaree, H. Haron, A. M. Abdulazeez, and S. R. M. Zeebaree, "Combination of k-means clustering with genetic algorithm: A review," Int. J. Appl. Eng. Res., vol. 12, no. 24, pp. 14238-14245, 2017.

[61] A. K. Jain, "Data clustering: 50 years beyond K-means," Pattern Recognit. Lett., vol. 31, no. 8, pp. 651-666, 2010, doi: 10.1016/j.patrec.2009.09.011.
[62] S. Hansen et al., "Unsupervised supervoxel-based lung tumor segmentation across patient scans in hybrid PET/MRI," Expert Syst. Appl., vol. 167, no. September 2020, 2021, doi: 10.1016/j.eswa.2020.114244.

[63] C. Series, "Detection of Alzheimer' s disease with segmentation approach using K- Means Clustering and Watershed Method of MRI image Detection of Alzheimer's disease with segmentation approach using K-Means Clustering and Watershed Method of MRI," 2021, doi: 10.1088/1742-6596/1725/1/012009.

[64] L. Rundo et al., "Tissue-specific and interpretable sub-segmentation of whole tumour burden on CT images by unsupervised fuzzy clustering," Comput. Biol. Med., vol. 120, no. April, p. 103751, 2020, doi: 10.1016/j.compbiomed.2020.103751.

[65] P. G. YILMAZ and G. ÖZMEN, "Follicle Detection for Polycystic Ovary Syndrome by using Image Processing Methods," Int. J. Appl. Math. Electron. Comput., vol. 8, no. 4, pp. 203-208, 2020, doi: 10.18100/ijamec.803400.

[66] I. H. Aboughaleb, M. H. Aref, and Y. H. El-Sharkawy, "Hyperspectral imaging for diagnosis and detection of ex-vivo breast cancer," Photodiagnosis Photodyn. Ther., vol. 31, no. April, p. 101922, 2020, doi: 10.1016/j.pdpdt.2020.101922.

[67] P. K. B. Rangaiah et al., "Clustering of Dielectric and Colour Profiles of an Ex-vivo Burnt Human Skin Sample," 14th Eur. Conf. Antennas Propagation, EuCAP 2020, 2020, doi: 10.23919/EuCAP48036.2020.9136005.

[68] H. Tai, M. Khairalseed, and K. Hoyt, "Adaptive attenuation correction during H-scan ultrasound imaging using K-means clustering," Ultrasonics, vol. 102, no. June, p. 105987, 2020, doi: 10.1016/j.ultras.2019.105987.

[69] R. Agrawal, M. Sharma, and B. K. Singh, "Segmentation of Brain Lesions in MRI and CT Scan Images: A Hybrid Approach Using kMeans Clustering and Image Morphology," J. Inst. Eng. Ser. B, vol. 99, no. 2, pp. 173-180, 2018, doi: 10.1007/s40031-018-0314-z.

[70] D. Borys, P. Bzowski, M. Danch-Wierzchowska, and K. PsiukMaksymowicz, "Comparison of k-means related clustering methods for nuclear medicine images segmentation," Ninth Int. Conf. Mach. Vis. (ICMV 2016), vol. 10341, no. Icmv 2016, p. 1034118, 2017, doi: $10.1117 / 12.2268825$.

[71] N. K. Student, "K-Means Clustering," pp. 1861-1865, 2017.

[72] P. Sarker, M. M. H. Shuvo, Z. Hossain, and S. Hasan, "Segmentation and classification of lung tumor from 3D CT image using K-means clustering algorithm," 4th Int. Conf. Adv. Electr. Eng. ICAEE 2017, vol. 2018-Janua, pp. 731-736, 2017, doi: 10.1109/ICAEE.2017.8255451.

[73] S. Yin, Y. Qian, and M. Gong, "Unsupervised hierarchical image segmentation through fuzzy entropy maximization," Pattern Recognit., vol. 68, pp. 245-259, 2017, doi: 10.1016/j.patcog.2017.03.012.

[74] J. Yu, D. Huang, and Z. Wei, "Unsupervised image segmentation via Stacked Denoising Auto-encoder and hierarchical patch indexing," Signal Processing, vol. 143, pp. 346-353, 2018, doi: 10.1016/j.sigpro.2017.07.009.

[75] A. Dharmarajan and T. Velmurugan, "Efficiency of k-Means and kMedoids Clustering Algorithms using Lung Cancer Dataset," Int. J. Data Min. Tech. Appl., vol. 5, no. 2, pp. 150-156, 2016, doi: 10.20894/ijdmta.102.005.002.011.

[76] A. A. Pravitasari et al., "Gaussian Mixture Model for MRI Image Segmentation to Build a Three-Dimensional Image on Brain Tumor Area," Matematika, vol. 36, no. 3, pp. 217-234, 2020, doi: 10.11113/matematika.v36.n3.1222.

[77] M. Kalra, M. Osadebey, N. Bouguila, M. Pedersen, and W. Fan, Online Variational Learning for Medical Image Data Clustering. 2020.

[78] K. Atrey, B. K. Singh, A. Roy, and N. K. Bodhey, "Breast cancer detection and validation using dual modality imaging," 20201 st Int. Conf. Power, Control Comput. Technol. ICPC2T 2020, pp. 454-458, 2020, doi: 10.1109/ICPC2T48082.2020.9071501. 